\title{
Numerical and Phenomenological Study of Desiccation of Soil
}

\author{
LiangBo Hu ${ }^{1}$, Hervé Péron ${ }^{2}$, Tomasz Hueckel ${ }^{1}$ and Lyesse Laloui ${ }^{2}$
}

\begin{abstract}
Desiccation cracks are assumed to be the consequence of an excess of tensile stresses induced by shrinkage of the drying body with a constrained kinematics. Free and constrained desiccation shrinking tests on silt samples are compared and simulated using linear hygro-elasticity. The results reveal diverse patterns of stress depending on the external constraints and presence of moisture gradient. The linear hygroelasticity captures well the drying process before the appearance of the cracks. For the tested material, the tensile axial stress at the first crack $(w=24 \%)$ was estimated at about $3.7 \mathrm{kPa}$.
\end{abstract}

\section{Introduction}

The objective of this paper is to explain some of the effects observed during drying of initially water saturated soils, and in particular the appearance of cracks. Cracking of soils leads invariably to deterioration of their mechanical and hydraulic properties. Assessment of the potential for such damage is difficult as mechanisms and principal variables in the process are poorly understood despite decades of research.

Drying has been investigated numerically by employing linear theories of hygroelasticity and moisture diffusion for rectangular slabs on either perfectly smooth or completely restraining rigid substrate, identifying principal mechanisms of their behavior during drying. Subsequently, these mechanisms have been investigated experimentally in simple drying tests on thin rectangular slabs of initially water saturated silt. The essential results of the experiments are first discussed, referring for a full recount of the testing techniques adopted to the previous papers of the authors

\footnotetext{
${ }^{1}$ Department of Civil and Environmental Engineering, Duke University, Durham, North Carolina. 27708, U.S.A. Email: liangbo.hu@duke.edu, hueckel@duke.edu, ${ }^{2}$ Soil Mechanics Laboratory, Ecole Polytechnique Fédérale de Lausanne (EPFL), Station 18, CH-1015 Lausanne, Switzerland. Email: herve.peron@epfl.ch, lyesse.laloui@epfl.ch.
} 
(Péron et al. 2005, 2006). These results are then discussed in the light of the simulations.

\section{Basic Concepts and Theoretical Mechanisms}

Hypotheses. Various authors have articulated to a different degree the main concept at the core of the current understanding of drying cracks (see e.g. Corte and Higashi 1960, Towner 1988). The loss of water due to evaporation is believed to cause shrinkage of soil via, most probably, a capillary mechanism. When shrinkage is constrained, reaction forces arise located at the constraints, which depend (presumably in a non-linear fashion) on the amount of shrinkage and deformability moduli. These reaction forces generate in turn stresses, which include tensile stress component. As tensile stresses grow with the progress of desiccation, they easily reach tensile strength. Cracks form at that point and propagate releasing a portion of the tensile stresses. Further shrinking induces a similar cycle with a newly configured geometry, i.e. cracked system, generating a new stress pattern, until tensile strength is reached again at new locations, yielding new cracks.

Several of the hypotheses in the above-described scenario require further elaboration and/or verification. First, it is known that shear and compressive strength evolve in the process of desaturation of soils, classically, as a function of suction. The evolution of tensile strength is much less understood. Second, development of the shrinkage strain and its relationship to water content change is nonlinear and it is not sure if all critical variables of the process have been identified. The patterns of stresses generated by the constrained shrinkage vary depending on the nature of the constraints themselves. Broadly speaking, the constraints may result from the external boundary conditions, kinematic compatibility, moisture content gradients, and internal boundary conditions resulting from the multi-phase structure of soil. The experiments discussed in this paper are aimed at the first two types of constraints.

Governing equations. Advantage is taken of direct analogy between equation describing transport of moisture in poro-elastic medium and its elastic response to changes in its moisture content, on the one hand, and thermo-elasticity of heat diffusing elastic medium on the other. In what follows we will limit our considerations to the linear, coupled theories. The diffusive water transfer is assumed to be governed by equation describing the change in water content $w$ (expressed in terms of \%):

$$
\frac{\partial w}{\partial}=\lambda \nabla^{2} w
$$

similar to heat conduction or mass diffusion law. Coefficient $\lambda$ is the diffusivity. The deformational response of the material is reproduced by linear hygro-elastic law. The law is expressed in terms of stress and strain tensors, respectively $\sigma_{i j}$, $\varepsilon_{k l}$. It includes an additional volumetric hydric strain, $\varepsilon^{h}$, proportional to water content change, hence

$$
\sigma_{i j}=D_{i j k l}\left(\varepsilon_{k l}-\varepsilon^{h} \delta_{k l}\right) ; \quad \varepsilon^{h}=\alpha \Delta w
$$


where $D_{i j k l}$ is the elastic stiffness tensor, depending on Young's modulus $E$, and Poisson's ratio $v$. The coefficient $\alpha$ is the hydric expansion/shrinkage strain coefficient, positive for shrinkage.

\section{Simulations}

Simulation assumptions. A 2D soil slab is examined, with the dimensions $L=20 \mathrm{~cm}$ and $H=2 \mathrm{~cm}$ at a uniform initial water content of $50 \%$. It is subjected to a uniform water content boundary condition and three kinds of kinematic boundary conditions: (i) a perfectly smooth substrate on which the slab is free to shrink unrestrained; (ii) a complete constraint at the bottom, preventing the slab from any sliding; (iii) a partial detachment of soil from the substrate (along a $5 \mathrm{~cm}$ segment at the slab extremity) allowing for a total freedom of displacement at this segment, while the other part of the bottom is constrained. The third configuration is included to simulate the edge curling effect. Moisture content at the boundaries evolves as follows: it starts at an initial value $w_{0}=50 \%$, decreasing with a constant rate of $1 \%$ per hour, over 50 hours.

For each configuration, two different simulations are performed to investigate the effect of water content gradient and the boundary constraints. A series of steady states with a homogeneous water content distribution is examined first to investigate the effect of kinematic constraints only. A diffusive transient water transfer case is studied next, originated from a uniform distribution of moisture and then following the above described history of the boundary moisture content. The goal is to examine the effect of water content gradient and a combination of both types of constraints.

Finite element simulations using FEAP code were performed in plane strain. The gravity is ignored in order to examine the shrinkage as isotropic and homogeneous. The material constants are: $E=1 \times 10^{5} \mathrm{~Pa} ; v=0.3$ ( $E$ and $v$ are deduced from triaxial test on Bioley silt), and $\alpha=5 \times 10^{-3} ; \lambda=0.122 \mathrm{~cm}^{2} / \mathrm{h}$ ( $\alpha$ and $\lambda$ are deduced from drying experiments described later).

Steady state with a uniform water mass loss. All the considered cases for the uniform moisture distribution are characterized by the stress fields related to the existing constraints. When there are no horizontal bottom constraints the deformation of the slab is a uniform contraction in all directions (Fig. 1a) with no stress. When the horizontal constraints at the bottom are imposed, a slight increase in the vertical shrinkage and a substantial decrease in the shrinkage of the central part of the slab are observed (Fig. 1b). They also generate horizontal reaction forces mainly at the extremities of the bottom, producing primarily tensile stresses. Maximum tensile stress is reached at the bottom corner, and otherwise is uniformly distributed over the rest of the slab, Fig. 2. Hence, in this scenario, the first crack would cause a detachment from the bottom corner, but could as well appear anywhere in the central part of the slab, as the differences in the stress values are not significant. The tensile stresses are generated to maintain strain compatibility conditions (see e.g. Kowalski 2003), in the absence of shear stresses prevented by the equilibrium conditions. The difference in shortening of the top versus the constrained bottom is confined to the vicinity of the edge, where it generates shear stresses. Hence, the top corner rises above the rest of the slab. 
For the partially supported slabs, intended to simulate specimen curling, the edge stress pattern observed in the fully constrained case is shifted toward the first support point, leaving the unsupported part virtually unstressed and unstrained. Consequently, the entire edge area becomes elevated as a rigid body above the original surface of the slab (Fig. 3). Hence, the entire curling effect is explained at the very basic level, with no moisture gradient, as due to bottom constraint induced shear effect. Maximum tensile stress also occurs near the point of the detachment of the slab bottom from the substrate, generating conditions for crack formation at that bottom point. However, to determine the location of such a point in reality, would require a more sophisticated analysis.
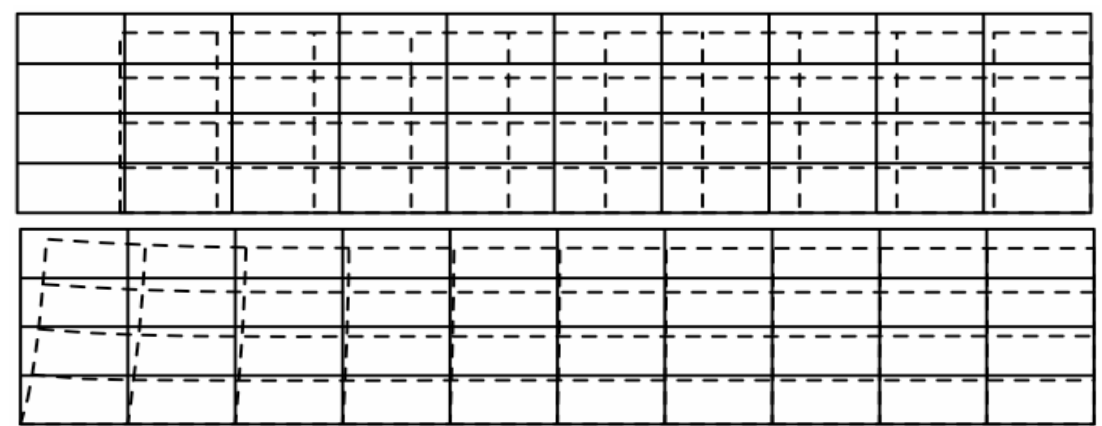

Figure 1. Deformation of the slab under (a) free and (b) constrained bottom conditions (half mesh). Solid line: initial configuration; dashed line: deformed mesh.

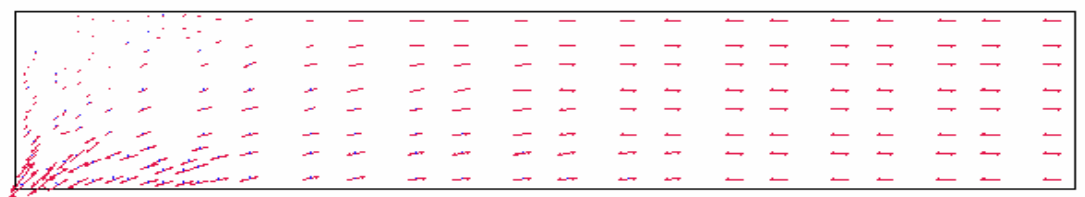

Figure 2. Principal stress pattern in the case of the fully constrained bottom (half mesh).

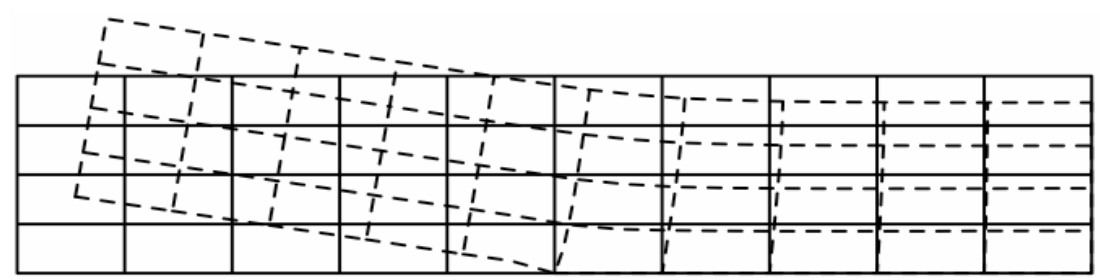

Figure 3. Deformation of the slab partially constrained at the bottom (half mesh)

Transient (gradiental) water content distribution. Taking into account the spatial progress of the moisture content change radically alters the pattern of the slab behavior. The resulting water content and stress distribution are shown in Fig. 4 (positive stress in tension). While deformation patterns do not dramatically differ from those in Figs.1 and 3, substantial horizontal stresses arise.

In the unconstrained bottom case, when water content drops to zero at the boundary (Fig. 4a) the horizontal stress takes a distribution like that of water content (Fig. 4b). Horizontal stresses are tensile at the surfaces and compressive in the middle, 
and self-equilibrated. In the case of the constrained bottom (Fig 4c), the horizontal stresses are all tensile and equilibrated by the bottom reactions. As a result, even for perfectly smooth substrate, almost a symmetric horizontal tensile stress reaches a maximum value at the surface, where it may cause cracking. Other stress components are negligible. For the constrained case, tensile stresses are slightly higher at the bottom near the corner, but otherwise their values at the bottom are only slightly higher than at the top. Finally, it must be emphasized that the moisture gradient is necessary (fast drying) for the occurrence of the stress distributions shown.
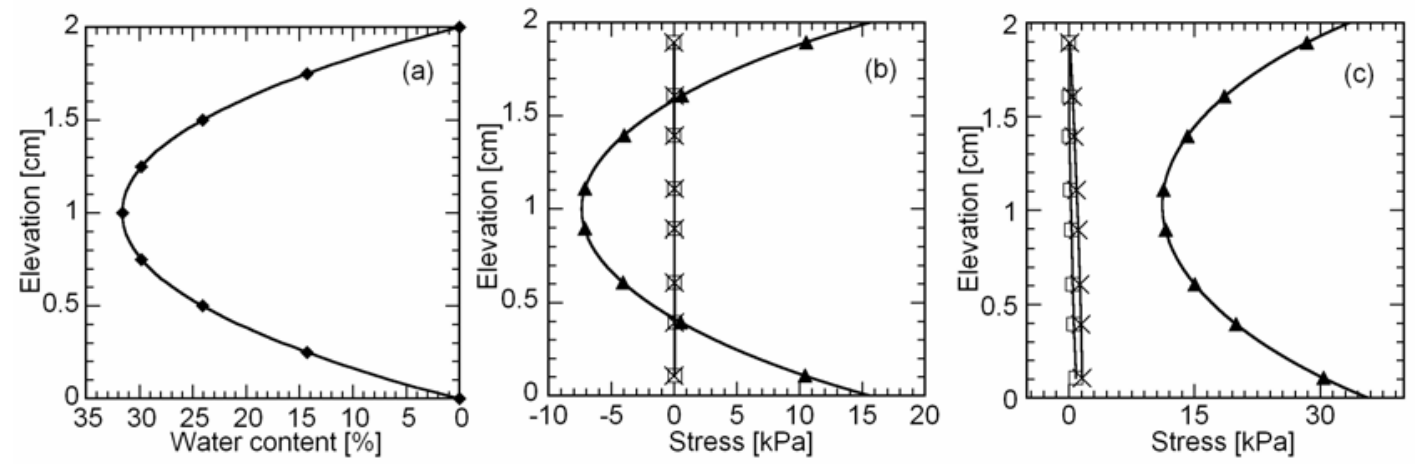

Figure 4. Water content (a) and stresses in free (b) and constrained (c) drying at $50 \mathrm{~h}$. $\boldsymbol{X}$ - shear stress; $\square$-vertical stress; $\boldsymbol{\Delta}$-axial stress.

\section{Assessment of the Experimental Results}

The material tested was Bioley silt, with a $27 \%$ clay fraction, at the initial water content of $49 \%$, which is around 1.5 times the liquid limit value. The soil was formed into thin rectangular slabs. Two batches of experiments were performed: on a Teflon substrate to model perfectly free shrinking; and on a grooved plate to model constrained bottom shrinking. Average gravimetric water content was measured by sample weighing, and strains in the three directions were measured with calipers (see Fig. 5). Details of the tests are reported elsewhere (Péron et al. 2005, 2006).

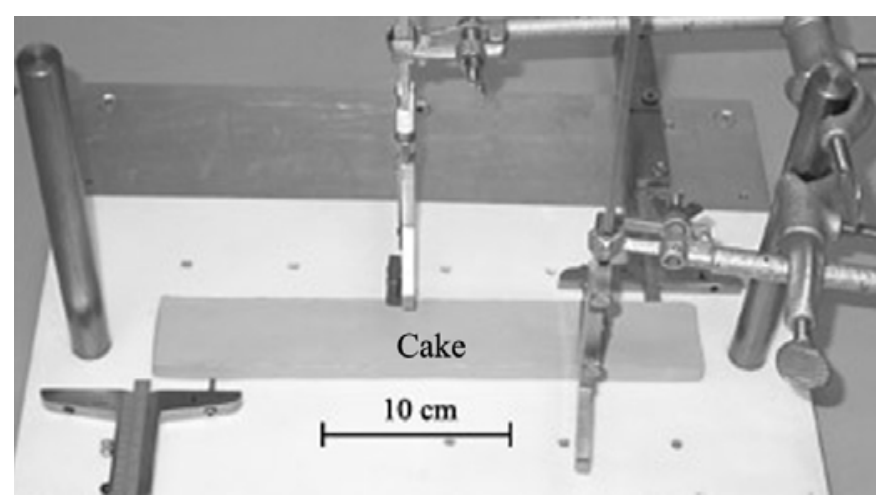

Figure 5. Set up for vertical and transversal strains.

In the case of free shrinking, two cases: very slow, and rapid dehydration were investigated. In the slow dehydration case, the slabs were dried in a controlled climate 
chamber. The rapid drying was conducted in an oven, taking advantage of the fact that thermal strains are of two orders of magnitude smaller than drying strains (Kowalski 2003). Only rapid drying leads to the development of cracks.
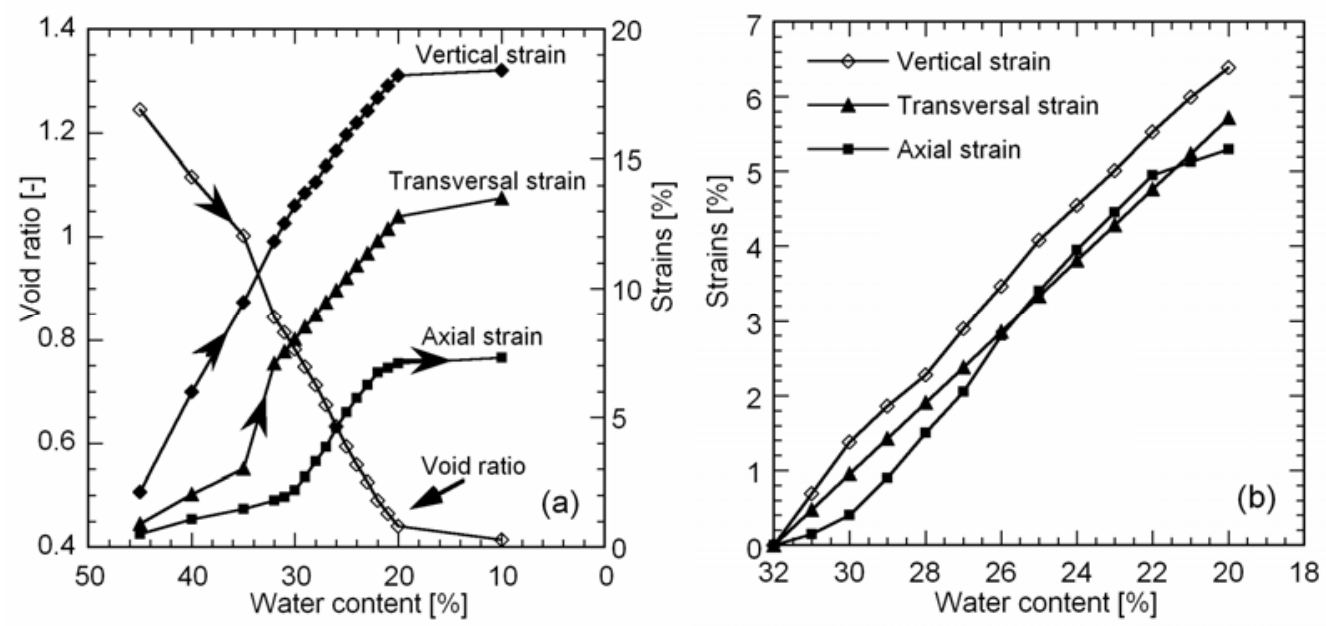

Figure 6. Unconstrained slab: (a) total strain and void ratio evolution, (b) strains for the second phase of drying.

In the case of slow drying, three phases of strain development were identified, as shown in Fig. 6a. The first phase $(35 \%<w<45 \%)$ is characterized by relatively low strain components parallel to the substrate, while the vertical strain shows quite an advanced shrinkage up to $w=18 \%$. In the second phase, all three strain components show a much higher rate vs. water content change, roughly the same as the vertical shrinkage in the previous phase. The third phase is characterized again by a very low strain change in all three directions. It is believed that only the second phase is the one of the uninhibited shrinkage, while the third (observed also widely in the past) characterizes a near-maximum grain packing configuration, with no space left for any further shrinkage. As for the constrained bottom shrinking tests, Bioley silt invariably cracks near the $23 \%$ strain threshold. The obtained crack patterns are shown in Fig. 7. It is hence considered that the second phase is representative as leading to cracking. The strains for this phase in unconstrained tests are redrawn in Fig. 6b. It is comforting to see that the strains are nearly isotropic in this phase. Additional tests are being conducted to clarify the nature of the phases one and three.

In the case of the constrained bottom shrinking, the strain components in the second phase (Fig. 8) are clearly different from those in the free shrinking. They are much higher in vertical direction than axially. This is in agreement with the results shown in Fig. 1b. To assess the character of the processes occurring in the constrained drying slabs, simulations were performed, this time in plane stress conditions (and matching the dimensions of the slabs). The first step was to calibrate the model, in particular to identify the value of the moisture shrinkage coefficient $\alpha$. It is believed that the second phase of the slow drying in the unconstrained case provides a reliable basis for it. The obtained value is $\alpha=0.0053$. Elasticity moduli used are as before. Two slabs were simulated using these parameters, respectively with the totally constrained and partially constrained bottom. The strain value was calculated dividing 
the average displacement of the extremities over the original dimension for the axial strain at the top corner, while for the vertical strain in the middle (Fig. 8 b). For the fully constrained case the numerical value of the axial strain is slightly above the experimental one, while it is much above that for the partially constrained case. The numerical values for the vertical strains are practically the same for both cases and are much higher than the axial ones, and are somewhat less than experimental ones.

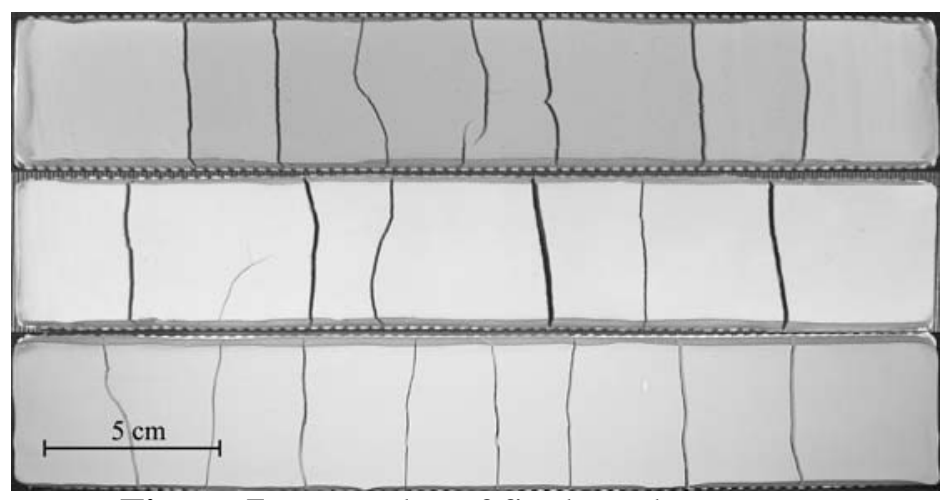

Figure 7. Examples of final crack patterns.
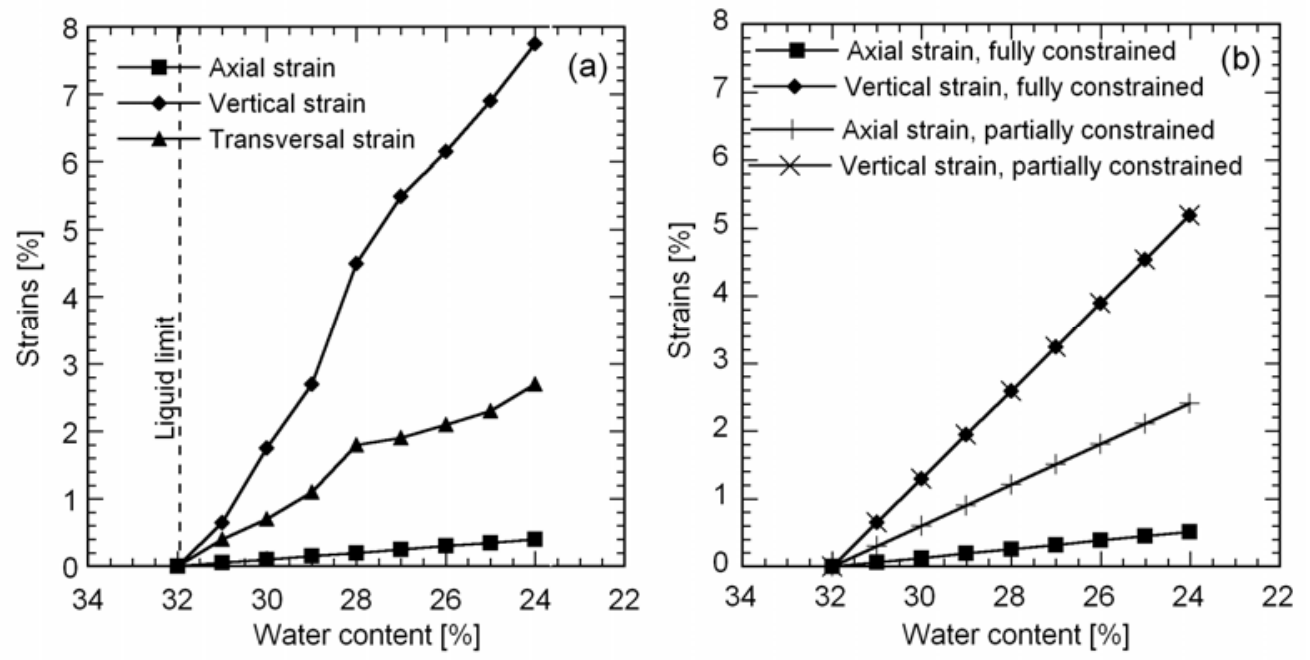

Figure 8. (a) Total strains evolution of the constrained slab, experimental results. (b) Total strains evolution of the fully and partially constrained slabs, simulation.

From the experiments it was also possible to calculate the purely mechanical strain values as a difference between the values attained in the constrained and unconstrained tests. Values corresponding to the first crack appearance at $w=24 \%$ for the vertical, axial and transversal strain components are respectively equal to: $-3.2 \%$, $3.6 \%$ and $1.03 \%$ (Péron et al. 2006). Substituting these values into a corresponding elasticity expression, the value of the axial stress at cracking was found as equal to $3.58 \mathrm{kPa}$. A similar value has been calculated from the above simulation (for fully constrained case) corresponding to the instant at $w=24 \%$, and resulted to be $3.9 \mathrm{kPa}$. The value obtained from the stress field in the simulation at the middle of the bottom of the slab is $3.92 \mathrm{kPa}$. These values are very close to the experimental ones, and may 
suggest the value of the tensile strength at that water content. Early estimates of the 1D tensile strength of Bioley silt at full saturation at $w=21 \%$, are of $5 \mathrm{kPa}$. Note that the transversal stress for the plane stress case is nearly the same as the vertical stress. When comparing these figures to the stress values in Figs $4 \mathrm{~b}$ and $\mathrm{c}$, note the difference in the total water content decrease, equal in the present case to $8 \%$ and up to $50 \%$ in the earlier example. As the elasticity solutions do not hold beyond the crack point, no assessment can be given to the appearance of the subsequent cracks.

\section{Conclusions}

The presented numerical study has been undertaken using a linear hygro-elasticity and linear moisture diffusion model, hence it does not simulate the behavior beyond the cracking onset. The extension into that area requires further progress in the modeling of the non-linear behavior in tensile stress range, and data regarding tensile strength evolution. The simulations also pertain to the behavior here referred to as the second phase of drying shrinkage. In the experiments the constraint at the bottom is not ideal, as some limited slippage of the slab on the substrate was observed. The observed phenomenon of edge curling was represented rather schematically, as if the point of the support edge was fixed, while in the tests it migrated. Nevertheless, it appears that the hygro-linear elasticity captures well the initial stage of the drying process in the considered experiment.

Acknowledgments. This work is funded by a cooperation of the US NSF, grant \# 0324543 and Swiss NSF, grant 200021-101917.

\section{References}

Corte, A. and Higashi, A. (1960). Experimental Research on Desiccation Cracks in Soil, Research Report 66: U.S. Army Snow Ice and Permafrost Res, Est, Wilmette, Ill.

Kowalski, S. J. (2003). Thermomechanics of Drying Processes. Springer, Berlin.

Péron, H., Laloui, L. and Hueckel, T. (2005). "An experimental evidence in desiccation cracking in sandy silt." Advanced Experimental Unsaturated Soil Mechanics, Tarantino et al., eds. Taylor \& Francis, London, 475-481.

Péron, H., Laloui, L., Hueckel, T. and Hu, L. (2006). "Experimental study of desiccation of soil." Proceedings of the Fourth International Conference on Unsaturated Soils, UNSAT 2006, in print.

Towner, G. D. (1988). "The influence of sand and silt size particles on the cracking during drying of small clay-dominated aggregates.” Journal of Soil Science 39, 347356. 